\title{
Parity-Violating $\Delta(1232)$ Electroweak Production: Axial Structure and New Physics
}

\author{
S.J. Pollock * \\ University of Colorado, Boulder CO 80309 \\ Nimai C. Mukhopadhyay \\ Rensselaer Polytechnic Institute, Troy, NY 12180 \\ M. Ramsey-Musolf \\ University of Connecticut, Storrs, CT 06260 \\ H.-W. Hammer \\ TRIUMF, 4004 Wesbrook Mall, Vancouver, B.C., Canada V6T $2 A 3$
}

J. Líu

\begin{abstract}
We consider prospects for studying the parity-violating electroweak excitation of the $\Delta(1232)$ resonance with polarized electron scattering [1]. We discuss the experimental feasibility and theoretical interpretability of such a measurement as well as the prospective implications for hadron structure theory. We also analyze the extent to which a PV $N \rightarrow \Delta$ measurement could constrain various extensions of the Standard Model.
\end{abstract}

If one measures a parity-violating (PV) asymmetry in electroproduction of the $\Delta(1232)$ using longitudinally polarized electrons on unpolarized nucleons, the resonant multipoles cancel in the ratio, leaving behind a relatively clean and simple structure. The $N \rightarrow \Delta$ transition is appealing for a PV experiment, since the $\Delta(1232)$ resonance is isolated from other nucleon resonances, the production cross section is relatively large, and the transition is pure isovector. This dominantly isovector nature eliminates uncertainties associated with the unknown strangeness content of the nucleon, and yields a unique sensitivity to possible contributions from additional heavy particles not appearing in the Standard Model (SM).

\footnotetext{
${ }^{*}$ Talk given at the ECT/CEBAF Workshop on $\mathrm{N}^{*}$ Physics and Nonperturbative QCD, Trento,
} May 1998. 
$N \rightarrow \Delta$ offers the additional advantage that it only couples strongly to one channel, $N \pi$. This allows one to treat unitarity issues quite rigorously [2].

In a recent paper [1], we examined this PV observable in some detail. The asymmetry has the following structure [1, 3, 3 : 4 :

$$
A_{L R}=\frac{N_{+}-N_{-}}{N_{+}+N_{-}}=\frac{-G_{\mu}}{\sqrt{2}} \frac{\left|q^{2}\right|}{4 \pi \alpha}\left[\Delta_{(1)}^{\pi}+\Delta_{(2)}^{\pi}+\Delta_{(3)}^{\pi}\right],
$$

where $N_{+}\left(N_{-}\right)$are the number of scattered electrons for a beam of positive (negative) helicity electrons, $q^{2}$ tells the square of the four-momentum transfer to the target; $\alpha$ and $G_{\mu}$ are the fine structure constant and the Fermi constant.

The quantities $\Delta_{(i)}^{\pi}$ denote the three primary contributions to the asymmetry discussed above. $\Delta_{(1)}^{\pi}=2\left(1-2 \sin ^{2} \theta_{W}\right)$ includes the entire resonant hadronic vector-current contribution to the asymmetry, at tree level in the SM. This term contains no dependence on hadronic form factors, owing to a cancellation between terms in the helicity-dependent and helicity-independent cross sections. The quantity $\Delta_{(2)}^{\pi}$ contains residual contributions from non-resonant, hadronic vector-current isoscalar backgrounds. The third term involves the axial-vector $N \rightarrow \Delta$ coupling: $\Delta_{(3)}^{\pi} \approx 2\left(1-4 \sin ^{2} \theta_{W}\right) F\left(q^{2}, s\right)$ (plus axial vector background contributions.) $F\left(q^{2}, s\right)$ involves a ratio of electroweak response functions, $s$ is the square of the total energy in the center of mass frame.

A precise measurement of $\Delta_{(1)}^{\pi}$ would provide a window on physics beyond the SM. We have found that a $1 \%$ knowledge of $\Delta_{(1)}^{\pi}$ would provide constraints roughly comparable to those presently obtained from atomic PV. A fairly demanding experimental setup might be able to achieve this level, if the non-resonant backgrounds and the axial contributions can both be understood at roughly $25-30 \%$ levels or better. The third term, $\Delta_{(3)}^{\pi}$, is extremely interesting from the standpoint of hadron structure. To a good approximation, the function $F\left(q^{2}, s\right)$ is proportional to the ratio of two transition form factors: $C_{5}^{A} / C_{3}^{V}$, where $V(A)$ correspond to the hadronic vector (axial vector) current. This ratio is the off-diagonal analog of the $G_{A} / G_{V}$ ratio in neutron $\beta$-decay. A measurement of $\Delta_{(3)}^{\pi}$ could correspondingly provide an opportunity to test low-energy consequences of chiral symmetry, such as the offdiagonal Goldberger-Treiman relation and its (small) chiral corrections [5]. In addition, a determination of $C_{5}^{A}$ could provide tests of lattice and quark model calculations and of the recipes proposed for correcting the vector form factor discrepancies.

The most serious uncertainties appear in two guises: (i) background contributions, contained in $\Delta_{(2)}^{\pi}$, and (ii) hadronic contributions to electroweak radiative corrections including two-boson exchange "dispersion corrections" and corrections induced by PV quark-quark interactions in the hadronic vertex. The former enter the analysis of all three of the $\Delta_{(i)}^{\pi}$, while the latter contribute to $\Delta_{(3)}^{\pi}$ only. Although an estimate of these hadronic PV corrections goes beyond the scope of the present work, we emphasize the importance of performing such an estimate when seeking to extract $C_{5}^{A} / C_{3}^{V}$ from $\Delta_{(3)}^{\pi}$.

For numerical estimates, we assume a plausible experimental scenario [6], 1000 hours of $100 \%$ polarized beam with a luminosity of $2 \times 10^{38} \mathrm{~cm}^{-2} \mathrm{~s}^{-1}$, solid angle $20 \mathrm{msr}$, energy range for the outgoing electrons $0.2 \mathrm{GeV}$. The figure of merit can easily be scaled for other assumptions. (For example, our solid angle is grossly conservative for backward angle experiments.) In Fig. 1a we show the $Q^{2}$-dependence of the axial term for several electron 
energies. We included a variety of models [1], and the theory spread is commensurate with the precision with which we anticipate one might realistically expect to determine the axial term. Consequently, a measurement of $A_{L R}(N \rightarrow \Delta)$ may only be marginally useful as a discriminator among models. Still, it would afford a determination of $C_{5}^{A} / C_{3}^{V}$ at the level of the experimental-theoretical discrepancies arising in the vector-current sector. More detailed numbers for various kinematics can be found in Table 1 . Fig. 1b shows the contributions of all three terms to the total asymmetry at forward angles. E.g., at $\theta=10^{\circ}$ and $\epsilon=1 \mathrm{GeV}$, we find $\Delta_{(2)}^{\pi} / \Delta_{(3)}^{\pi} \approx 6 \%$, and so even a large uncertainty in $\Delta_{(2)}^{\pi}$ has negligible effect on an extraction of the axial term.

In our calculations, we use a recent model-dependent estimate of the background [7]. At kinematics well suited for a determination of $\sin ^{2} \theta_{W}$, the vector-current background contributes about $4-6 \%$ of the total. Thus, a probe for new physics at the $1 \%$ level would require a theoretical uncertainty in the background to be no more than $15-25 \%$ of the total for $\Delta_{(2)}^{\pi}$. Since the present model permits a $50 \%$ uncertainty in the background and still produces agreement with inclusive EM pion production data, one could argue that a model estimate of $\Delta_{(2)}^{\pi}$ is not sufficient for purposes of undertaking a $1 \%$ SM test. It appears that a model-independent approach with an experimental isospin decomposition of the EM pion production process offers the best hope for eliminating vector-current background uncertainties.

In summary, we have analyzed the PV $N \rightarrow \Delta$ transition asymmetry. We considered the sensitivity of $A_{L R}$ to various scenarios for physics beyond the SM - such as leptoquarks, additional neutral gauge bosons, and fermion compositeness - as well as to transition form factors of interest to hadron structure theory. After estimating the precision with which $A_{L R}$ might be determined in a realistic experiment, we estimated the scale of background effects. The use of $A_{L R}(N \rightarrow \Delta)$ as a probe of hadron structure appears to be a feasible prospect at present. $\mathrm{A} \sim 25 \%$ determination of the hadronic axial vector response could be carried out with realistic running times. At reasonable kinematics for such a measurement, the backgrounds appear to be sufficiently under control. While a $25 \%$ determination of $C_{5}^{A} / C_{3}^{V}$ would not allow for a detailed discrimination among model predictions, it would significantly improve upon knowledge from charged current neutrino reactions and test model predictions at the level of the theoretical-experimental discrepancies arising in the vector current sector. A complete theoretical analysis of $\Delta_{(3)}^{\pi}$, including effects of potentially large and uncertain radiative corrections associated with hadronic PV, awaits a future study.

\section{ACKNOWLEDGMENTS}

The work was supported in part by the U.S. Department of Energy. Thanks also to the ECT/CEBAF for their partial support at this workshop. 


\section{REFERENCES}

[1] N.C. Mukhopadhyay, M.J. Ramsey-Musolf, S. Pollock, J. Liu, and H.-W. Hammer. Nuclear Physics A633 (1998) 481. (nucl-th/9801025)

[2] R. M. Davidson and N. C. Mukhopadhyay, Phys. Rev. D42, 20 (1990); R. M. Davidson, N. C. Mukhopadhyay, and R. S. Wittman, ibid., D43, 71 (1991).

[3] M. J. Musolf et al., Phys. Rep. 239, 1 (1994).

[4] D. R. T. Jones and S. T. Petcov, Phys. Lett. 91B, 137 (1980), R. N. Cahn and F. J. Gilman, Phys. Rev. D17, 1313 (1978),

[5] T. R. Hemmert, B. R. Holstein, and N. C. Mukhopadhyay, Phys. Rev. D51, 158 (1995).

[6] Jefferson Laboratory experiment \# E97-104, "Measurement of the Parity Violating Asymmetry in the N to Delta Transition", S.P. Wells, N. Simicevic, and K. Johnston, spokespersons (1997).

[7] H. -W. Hammer and D. Drechsel, Z. Phys. A353, 321 (1995) 


\section{TABLES}

\begin{tabular}{|ccc|ccccc|}
\hline$E(\mathrm{GeV})$ & $\theta_{\mathrm{lab}}\left({ }^{o}\right)$ & $Q^{2}\left(\mathrm{GeV}^{2}\right)$ & $10^{5} A_{\text {tot }}$ & $\frac{\delta A_{\text {stat }}}{A_{\text {tot }}}(\%)$ & $\frac{\Delta_{(2)}^{\pi}}{\Delta^{\pi}}(\%)$ & $\frac{\Delta_{(3)}^{\pi}}{\Delta^{\pi}}(\%)$ & $\frac{A_{(3)}}{\delta A_{\text {stat }}}$ \\
\hline .5 & 10. & .002 & -.03 & 45.9 & -.24 & 20.4 & .4 \\
1.0 & 10. & .020 & -.22 & 5.9 & -.7 & 11.0 & 1.9 \\
4.0 & 10. & .418 & -4.17 & .7 & -5.5 & 2.6 & 4.0 \\
\hline .5 & 90. & .106 & -1.24 & 10.9 & -2.81 & 17.1 & 1.6 \\
1.0 & 90. & .641 & -6.79 & 5.2 & -6.4 & 8.3 & 1.6 \\
4.0 & 90. & 5.566 & -54.46 & 26.8 & -13.4 & .8 & .0 \\
\hline .5 & 180. & .157 & -1.81 & 11.6 & -3.70 & 15.5 & 1.3 \\
1.0 & 180. & .846 & -8.85 & 7.5 & -7.0 & 7.1 & .9 \\
4.0 & 180. & 6.150 & -60.08 & 43.8 & -10.4 & .6 & .0 \\
\hline
\end{tabular}

TABLE I. Our estimates of $A_{L R}$, experimental statistical uncertainty for $A_{L R}$ (given our assumptions), vector-current backgrounds, contribution of axial multipoles, and ratio of axial contribution to statistical uncertainty, respectively, as functions of electron energy and scattering angle.

$Q^{2}$ is calculated assuming we are on the $\Delta$ peak. $\Delta^{\pi}=\Delta_{(1)}^{\pi}+\Delta_{(2)}^{\pi}+\Delta_{(3)}^{\pi}, A_{(3)}$ is the contribution to the asymmetry arising from $\Delta_{(3)}^{\pi}$. 


\section{FIGURES}
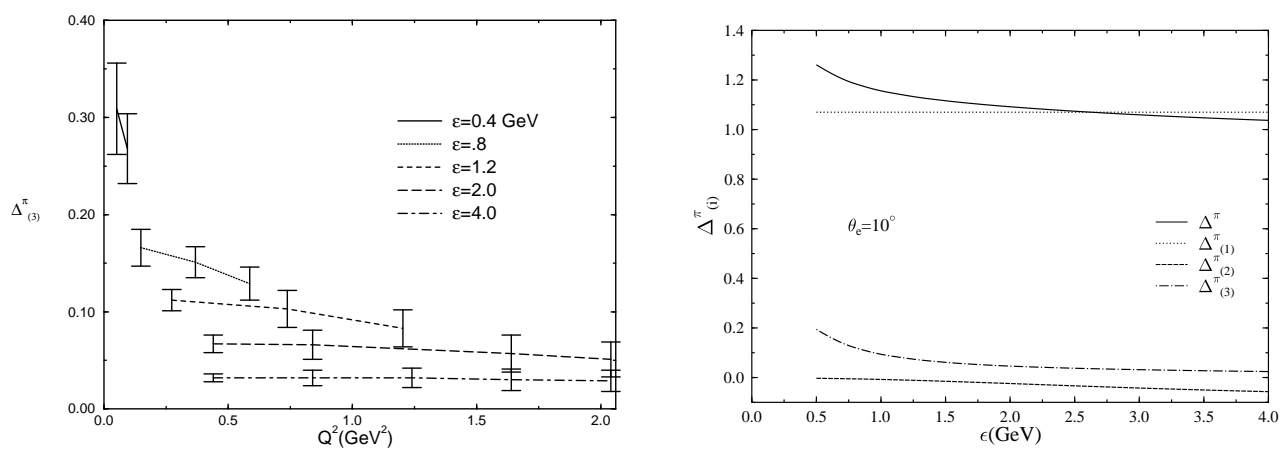

FIG. 1. a) $\Delta_{(3)}^{\pi}$ as a function of $Q^{2}$ for different incident electron energies, $\epsilon$. Error bars show a rough spread of theoretical results. b) Contributions to the asymmetry from the $\Delta_{(i)}^{\pi}$ as a function of incident energy for $\theta=10^{\circ}$. Here, $\Delta^{\pi}=\Delta_{(1)}^{\pi}+\Delta_{(2)}^{\pi}+\Delta_{(3)}^{\pi}$.

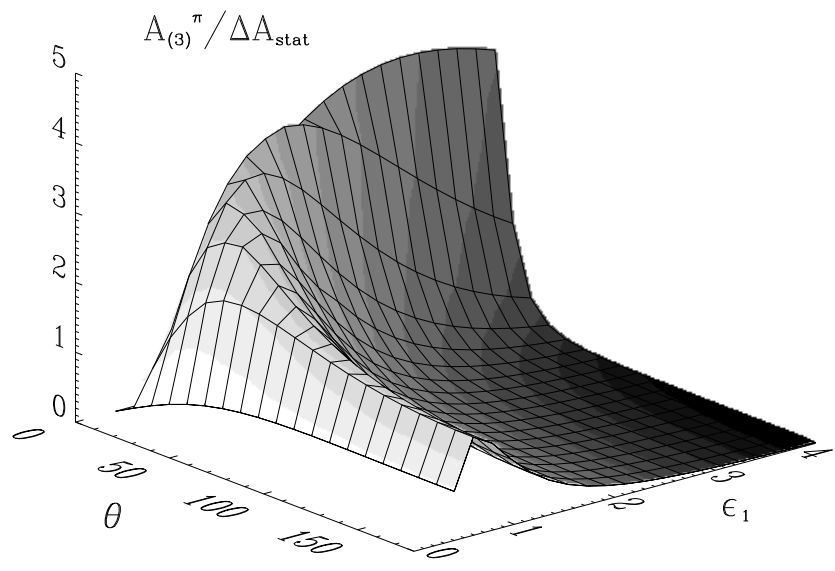

FIG. 2. A 3-D plot of $\frac{A_{(3)} / A_{\text {tot }}}{\delta A_{\text {stat }} / A_{\text {tot }}}$, versus both incident energy and electron scattering angle. (The shading is determined by the value of $\Delta_{(3)}^{\pi}$, smaller values are shaded darker) 